\title{
Expressão dos caracteres e seleção de clones de batata nas condições de cultivo de primavera e outono
}

\author{
Trait expression and selection of potato clones in the spring and autumn growth conditions
}

\author{
Douglas Renato MüllerI Dilson Antônio Bisognin'I* Jerônimo Luiz AndrioloII \\ Glademir Roque Morin Junior ${ }^{\mathrm{III}}$ Francisco Saccol Gnocato ${ }^{\mathrm{III}}$
}

RESUMO

No Rio Grande do Sul, a batata é cultivada na primavera e no outono, período em que as condições contrastantes de cultivo podem afetar os teores de matéria seca, açúcares redutores, amido e amilose e a cor de chips. O objetivo deste trabalho foi avaliar o efeito das condições de cultivo de primavera e outono na expressão dos caracteres de qualidade de processamento, visando desenvolver estratégias de melhoramento genético que maximizem o ganho de seleção e facilitem a identificação de clones de batata com potencial para processamento. O experimento foi conduzido em um esquema fatorial com 21 clones e duas épocas de cultivo, no delineamento inteiramente casualizado, com três repetições. Foram avaliados os teores de matéria seca, açúcares redutores, amido e amilose e a cor de chips dos tubérculos produzidos na primavera de 2006 e no outono de 2007. As condições de cultivo de primavera maximizaram o ganho genético de seleção para açúcares redutores, cor de chips, amido e amilose. Os ganhos de seleção para matéria seca foram similares em ambos os cultivos. As condições ambientais distintas na primavera $e$ no outono afetaram a seleção e resultaram em diferentes grupos de clones superiores. Os clones SMA508-2, SMA508-4 e SMA519-1 apresentaram, em ambas as condições de cultivo, uma combinação de caracteres de qualidade de processamento equilibrada e superior à melhor testemunha. A seleção de clones para processamento industrial deve ser realizada no cultivo de primavera, quando as condições ambientais são mais favoráveis para a expressão dos caracteres de qualidade de tubérculo no Rio Grande do Sul.

Palavras-chave: Solanum tuberosum L., matéria seca, açúcares redutores, amido, amilose, cor de chips.

\begin{abstract}
Potato grows during spring and autumn conditions in Rio Grande do Sul, Brazil. These contrasting conditions may affect dry matter, reduced sugars, starch and amylase contents and chip color. The aim of this work was to evaluate the effect of spring and fall growth conditions in the expression of processing quality traits to develop breeding strategies to maximize the selection gain and facilitate the identification of potato clones with high processing potential. The experiment was a factorial of 21 clones and two growth seasons in the complete random design, with three replications. Tubers were produced during spring 2006 and fall 2007 growth seasons and evaluated for dry mass, reduced sugars, starch and amylase contents and chip color. Spring growth conditions maximized the gain from selection for reduced sugars, chip color, starch and amylase. Similar selection gain for dry mass was found in spring and fall seasons. The distinct environmental conditions of spring and fall did affect the selection gain and resulted in different groups of superior clones. The clones SMA508-2, SMA508-4 and SMA519-1 showed the best combination of processing quality traits in both growing conditions and higher than the best check. Clone selection for processing quality should be done in spring season, when environmental conditions improve the expression of the processing quality traits in Rio Grande do Sul State.
\end{abstract}

Key words: Solanum tuberosum L., dry matter, reduced sugars, starch, amylase, chip color.

\section{INTRODUÇÃO}

Devido a sua versatilidade gastronômica e tecnológica, a batata (Solanum tuberosum L.) é um

IPrograma de Pós-graduação em Agronomia, Universidade Federal de Santa Maria (UFSM), 97105-900, Santa Maria, RS, Brasil.

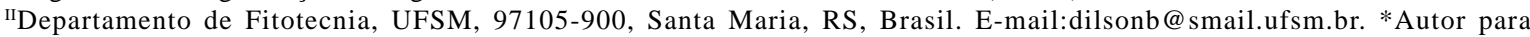
correspondência.

IIICurso de Agronomia, UFSM, Santa Maria, RS, Brasil. 
dos alimentos mais consumidos no mundo, podendo ser utilizada na forma de flocos e granulados, de fritas à francesa e chips, pré-cozidas e congeladas ou cozidas e enlatadas (COELHO et al., 1999). A falta de matériaprima de qualidade e em quantidades adequadas tem levado a indústria de processamento à importação de batata pré-frita e congelada. Esse mercado poderia ser suprido por cultivares de alta qualidade de processamento adaptadas a condições brasileiras de cultivo, o que justifica a ampliação de pesquisas na área de melhoramento genético de batata.

Dentre os caracteres que influenciam a qualidade de tubérculos para a industrialização, os teores de matéria seca e de açúcares redutores estão associados ao rendimento e à qualidade do produto processado, por determinar a absorção de gordura durante a fritura, a textura e o sabor do produto final (SILVA, 1991; FELTRAN, et al., 2004). Tubérculos destinados ao processamento na forma de chips devem apresentar teores de matéria seca superiores a $20 \%$ para uma boa qualidade das fritas produzidas (BRODY, 1969; OLIVEIRA et al., 2006). No entanto, o teor de matéria seca é influenciado pela cultivar, pelas condições de cultivo e pelo grau de maturidade dos tubérculos (MELO, 1997), ou seja, a qualidade aumenta com a maturidade dos tubérculos.

Os açúcares redutores (glicose e frutose) servem como substrato para a Reação de Maillard, que ocorre durante a fritura e portanto, em altos níveis, resultam na produção de chips de cor escura e gosto amargo (COELHO et al., 1999; FELTRAN et al., 2004). O teor de açúcares redutores é o resultado de vários fatores ambientais que ocorrem durante o crescimento e desenvolvimento dos tubérculos (HERTOG et al., 1997), sendo os teores ideais para processamento na forma de chips entre 10 e $15 \mathrm{mg}$ g de matéria seca- ${ }^{-1}$ (ZORZELLA et al., 2003). O teor de açúcares redutores, por afetar a cor e o gosto, é um fator determinante para a comercialização do produto processado. Existem vários métodos que são utilizados para classificar a cor de chips, como o sistema tridimensional de cores CIE (L*a*b*) proposto por COLEMAN (2004), cuja cor de chips após a fritura com $\mathrm{L}^{*}<55$ é inaceitável, $L^{*} \geq 55$ e $\leq 70$ é aceitável e $L^{*}>70$ é de alta qualidade.

O amido constitui-se na principal fonte de reserva dos tubérculos de batata, representando entre 60 e $80 \%$ da matéria seca (FONTES \& FINGER, 2000; FREITAS et al., 2006). A deposição dos grãos de amido nos amiloplastos decorre da transformação da sacarose, glicose e maltose em amilose e amilopectina (FONTES \& FINGER, 2000), que são os dois principais constituintes do amido. Em geral, o amido da batata apresenta $20 \%$ de amilose e $80 \%$ de amilopectina
(LEONEL, 2005). Alto teor de amilose é desejado quando o produto necessita ser crocante e resistente (FRANCO et al., 2002), como é o caso do processamento na forma de chips, cujos teores variam com a cultivar e as condições de cultivo (FRANCO et al., 2002; NODA et al., 2004).

No Rio Grande do Sul, a batata é cultivada na primavera e no outono, período em que as condições de cultivo podem afetar os teores de matéria seca, açúcares redutores, amido e amilose e a cor de chips. Portanto, o objetivo deste trabalho foi avaliar o efeito das condições de cultivo de primavera e outono na expressão dos caracteres de qualidade de processamento, visando desenvolver estratégias de melhoramento genético que maximizem o ganho de seleção e facilitam a identificação de clones de batata com potencial para processamento industrial.

\section{MATERIAL E MÉTODOS}

Os tubérculos foram produzidos em campo na Fundação Estadual de Pesquisa Agropecuária (FEPAGRO), em Júlio de Castilhos, Rio Grande do Sul (RS). No cultivo de primavera, o plantio foi realizado em 23 de agosto, e a colheita foi realizada em 19 de dezembro de 2006. No outono, o plantio foi realizado em $1^{\circ}$ de março, e a colheita foi realizada em 7 de junho de 2007. Os tratos culturais e o manejo da lavoura foram feitos de acordo com as recomendações técnicas para o cultivo da batata (BISOGNIN, 1996). Dez dias antes da colheita foi realizada a dessecação da parte aérea com Paraquat.

Após a colheita, os tubérculos foram transportados para o Departamento de Fitotecnia da Universidade Federal de Santa Maria (UFSM) e submetidos ao processo de cura, que consistiu no armazenamento a $20^{\circ} \mathrm{C}$ por 15 dias. Após esse período, foram determinados os teores de matéria seca, açúcares redutores, amido e amilose e a cor de chips. O experimento foi conduzido em um fatorial de 21 clones (SMA501-1, SMA502-1, SMA503-1, SMA504-2, SMA505-2, SMA505-3, SMA505-7, SMA506-4, SMA508-2, SMA508-4, SMA513-2, SMA514-8, SMA514-10, SMA514-11, SMA516-2, SMA517-2, SMA517-3, SMA519-1, SMA520-5, SMIJ461-1 e Asterix) e duas épocas de cultivo (primavera e outono), no delineamento inteiramente casualizado, com três repetições. A cultivar 'Asterix', muito cultivada no Rio Grande do Sul, e o clone SMIJ461-1, com conhecido potencial para processamento na forma de chips (FREITAS et al., 2006), foram utilizados como testemunhas. Para fins de simplificação, a cultivar ‘Asterix’ será referida simplesmente como clone. 
O teor de matéria seca foi determinado por meio do acondicionamento das amostras de batata, previamente descascadas e picadas, em estufa à temperatura de $60^{\circ} \mathrm{C}$ até obter massa constante. Os teores de açúcares redutores e amido foram determinados conforme a metodologia de LONG \& CHISM (2004) e adaptações propostas por FREITAS et al. (2006). O teor de amilose foi quantificado por meio da medida da absorbância do complexo amidoiodo (GILBERT \& SPRAGG, 1964), baseado na capacidade da estrutura helicoidal que a amilose tem em formar complexos de inclusão com o iodo. A cor de chips foi determinada em três amostras de três tubérculos, das quais foram utilizadas três fatias transversais e centrais de $2 \mathrm{~mm}$ de espessura de cada tubérculo. As nove fatias foram fritas em fritadeira industrial a gás (Top Taylor, modelo TTF-35-G), utilizando gordura vegetal hidrogenada na temperatura de $185^{\circ} \mathrm{C}$, controlada por termostato, até cessar a borbulha. Cada amostra foi submetida a duas medidas de coloração (colorímetro Minolta, modelo CR310) do sistema CIE ( $\mathrm{L}^{*} \mathrm{a} * \mathrm{~b} *$ ), sendo considerados os valores medidos na escala $\mathrm{L}$, que varia do preto ao branco (COLEMAN, 2004).

Os dados foram submetidos à análise da variância, e as médias de clones foram comparadas pelo teste de Scott-Knott e de época de cultivo pelo teste de Tukey, a 5\% de probabilidade de erro. A identificação dos melhores clones foi realizada com base na soma de postos proposta por MULAMBA \& MOCK (1978) e descrita por CRUZ \& REGAZZI (1997). Esse índice consiste em classificar os clones em relação a cada um dos caracteres, em ordem favorável ao melhoramento (açúcares redutores com o menor valor e os demais caracteres com o maior valor). A ordem de classificação de cada clone para todos os caracteres avaliados foi somada, resultando em um valor tomado como índice para a seleção dos clones (CRUZ \& REGAZZI, 1997). Os clones com índices menores do que a melhor testemunha (Asterix ou SMIJ461-1) foram selecionados. O ganho de seleção foi calculado pela diferença entre a média dos clones selecionados e a média de todos os clones avaliados, para os cinco caracteres avaliados e representado em percentagem.

\section{RESULTADOS E DISCUSSÃO}

A análise da variância mostrou diferenças significativas $(\mathrm{P}<0,05)$ para a interação entre clones e época de cultivo para todas as variáveis avaliadas (Tabelas 1 e 2). Os clones SMA513-2 e SMA519-1, em ambos os cultivos, e SMA501-1, SMA514-8, SMA5205 e SMA505-3, no cultivo de outono, apresentaram teores de açúcares redutores menores do que 15mg g de matéria seca-1 ${ }^{-1}$ considerados como aceitáveis para processamento industrial (ZORZELLA et al., 2003) (Tabela 1). A época de cultivo não afetou o teor de açúcares redutores dos clones SMA513-2, SMA519-1, SMA503-1, SMA501-1, SMA514-10, SMA517-3, SMIJ461-1, Asterix, SMA516-2, SMA514-8, SMA5172, SMA514-11 e SMA508-4, o que é importante quando se buscam clones com potencial de processamento em ambas as condições de cultivo. Entretanto, quando o objetivo é a seleção de clones para o processamento na forma de chips, essa menor sensibilidade deve vir acompanhada de baixos teores de açúcares redutores, como é o caso dos clones SMA513-2 e SMA519-1, que, além de não terem sido afetados pelas condições de cultivo de primavera e outono, também apresentaram teores aceitáveis pela indústria de processamento.

Quando considerados os teores de açúcares redutores entre cultivos, observou-se, nos clones SMA508-2 e SMA506-4, maior teor em tubérculos produzidos no outono. Nos clones SMA520-5, SMA505-3, SMA505-2, SMA504-2, SMA502-1 e SMA505-7, o teor mais elevado foi observado na primavera. Uma das hipóteses que tem sido apresentada para explicar o maior teor de açúcares redutores é o estado de maturação dos tubérculos no momento da colheita (MELO, 1997). Devido às condições ambientais no cultivo de outono, o número de tubérculos ainda imaturos no momento da colheita é maior nessa época, os quais apresentam teor mais elevado de açúcares redutores do que aqueles colhidos maduros. Entretanto, essa hipótese não se ajusta para todos os clones que foram avaliados neste trabalho. Essa diferença entre clones poderia estar relacionada à origem genética. Enquanto os clones SMA508-2 e SMA506-4 são o resultado de cruzamento envolvendo parentais mais bem adaptados às condições de cultivo da região central do Rio Grande do Sul (Asterix e SMINIAIporã, respectivamente), todos os demais clones são resultantes de cruzamentos entre genitores oriundos de regiões temperadas e, portanto, menos adaptados às condições locais de cultivo. A maioria dos clones, no entanto, não apresentou diferença entre cultivos, o que é uma característica importante em regiões onde o cultivo da batata é feito em épocas com condições ambientais contrastantes, demonstrando menor efeito ambiental sobre o teor dos açúcares redutores.

A cor de chips, influenciada principalmente pelo teor de açúcares redutores (FELTRAN et al., 2004), é um fator determinante para a comercialização do produto processado. Com exceção do clone SMA5021, na primavera, e do clone SMA513-2, no outono, todos 
Tabela 1 - Teor de açúcares redutores na matéria seca, cor de chips e teor de matéria seca de clones de batata produzidos na primavera e no outono. Santa Maria, RS, 2007.

\begin{tabular}{|c|c|c|c|c|c|c|}
\hline \multirow{2}{*}{ Clones } & \multicolumn{2}{|c|}{ Açúcares redutores (mg g de $\mathrm{MS}^{-1}$ ) } & \multicolumn{2}{|c|}{--------Cor de chips $\left(\mathrm{L}^{* 1}\right)$---------- } & \multicolumn{2}{|c|}{---------Matéria seca (\%)---------- } \\
\hline & Primavera & Outono & Primavera & Outono & Primavera & Outono \\
\hline SMA513-2 & $11,57 \mathrm{~A} \mathrm{a}^{2}$ & $12,38 \mathrm{~A} \mathrm{~b}$ & $66,40 \mathrm{~A} \mathrm{a}$ & $72,62 \mathrm{~A} \mathrm{a}$ & $25,26 \mathrm{~A} \mathrm{~b}$ & 19,77 B c \\
\hline SMA519-1 & $13,10 \mathrm{~A} \mathrm{a}$ & 9,33 A а & $68,50 \mathrm{~A} \mathrm{a}$ & $66,75 \mathrm{~A}$ c & $26,27 \mathrm{~A} \mathrm{a}$ & $22,93 \mathrm{~B} \mathrm{a}$ \\
\hline SMA508-2 & 17,41 B b & 39,83 A & $66,49 \mathrm{~A} \mathrm{a}$ & 67,61 A с & $25,11 \mathrm{~A} \mathrm{~b}$ & $23,16 \mathrm{~A} \mathrm{a}$ \\
\hline SMA503-1 & $18,05 \mathrm{~A} \mathrm{~b}$ & $23,65 \mathrm{~A} \quad \mathrm{e}$ & 64,57 A b & 66,98 A с & $23,34 \mathrm{~A}$ c & $21,10 \mathrm{~A} \mathrm{~b}$ \\
\hline SMA501-1 & 18,38 A b & 9,35 A a & $62,68 \mathrm{~A} \mathrm{~b}$ & 66,91 A с & $24,46 \mathrm{~A} b$ & 21,64 B b \\
\hline SMA506-4 & 19,18 B b & 37,19 A & $66,06 \mathrm{~A} \mathrm{a}$ & 65,97 A c & $21,51 \mathrm{~A} \quad \mathrm{e}$ & 19,29 A c \\
\hline SMA514-10 & 20,57 A b & $30,38 \mathrm{~A} \quad \mathrm{~g}$ & 63,32 A b & $65,14 \mathrm{~A} \quad \mathrm{c}$ & $25,68 \mathrm{~A} \mathrm{a}$ & $22,02 \mathrm{~B} \mathrm{a}$ \\
\hline SMA517-3 & 22,43 A c & $16,94 \mathrm{~A} \quad \mathrm{c}$ & 58,41 A c & $59,69 \mathrm{~A} \quad \mathrm{e}$ & $21,45 \mathrm{~A} \quad \mathrm{e}$ & $17,21 \mathrm{~B} \quad \mathrm{~d}$ \\
\hline SMIJ461-1 & 22,77 A c & 18,67 A c & 61,72 B c & 68,56 B b & $23,56 \mathrm{~A} \quad \mathrm{c}$ & 21,39 A b \\
\hline Asterix & 23,10 A c & $19,77 \mathrm{~A} \quad \mathrm{~d}$ & 59,91 A с & 64,88 A c & 23,07 A c & 19,35 B c \\
\hline SMA516-2 & $23,80 \mathrm{~A} \quad \mathrm{c}$ & $25,92 \mathrm{~A} \quad \mathrm{f}$ & $55,87 \mathrm{~B} \quad \mathrm{~d}$ & 65,41 A c & 23,16 A c & 20,18 B C \\
\hline SMA514-8 & 24,73 A c & 13,78 A b & 62,55 B b & 69,99 A b & $22,76 \mathrm{~A} \quad \mathrm{~d}$ & $22,06 \mathrm{~A} \mathrm{a}$ \\
\hline SMA517-2 & 25,49 A c & 18,15 A c & 60,42 A $\mathrm{c}$ & 65,98 A c & $23,61 \mathrm{~A} \quad$ с & $21,56 \mathrm{~A} \mathrm{~b}$ \\
\hline SMA514-11 & $26,94 \mathrm{~A} \quad \mathrm{~d}$ & 17,84 A c & $68,13 \mathrm{~A} \mathrm{a}$ & 67,67 A c & 24,79 A b & 20,48 B b \\
\hline SMA520-5 & $28,85 \mathrm{~A} \quad \mathrm{~d}$ & $10,66 \mathrm{~B} \mathrm{a}$ & 61,29 B c & 66,70 A с & 24,89 A b & 21,15 B b \\
\hline SMA508-4 & 28,94 A & 35,96 A & $68,88 \mathrm{~A} \mathrm{a}$ & $68,42 \mathrm{~A} \mathrm{~b}$ & $25,44 \mathrm{~A} \mathrm{a}$ & $22,33 \mathrm{~B} \mathrm{a}$ \\
\hline SMA505-3 & $34,82 \mathrm{~A}$ & 13,86 B b & $57,66 \mathrm{~A} d$ & $63,57 \mathrm{~A} d$ & $19,12 \mathrm{~A}$ & 20,89 A b \\
\hline SMA505-2 & $34,92 \mathrm{~A}$ & $19,99 \mathrm{~B} \quad \mathrm{~d}$ & $63,42 \mathrm{~A} \quad \mathrm{~b}$ & $65,14 \mathrm{~A} \quad \mathrm{c}$ & $22,28 \mathrm{~A} \quad \mathrm{~d}$ & $21,03 \mathrm{~A} \mathrm{~b}$ \\
\hline SMA504-2 & $40,18 \mathrm{~A}$ & $26,73 \mathrm{~B} \quad \mathrm{f}$ & $57,12 \mathrm{~A} d$ & $58,06 \mathrm{~A} \quad \mathrm{e}$ & $20,88 \mathrm{~A} \quad \mathrm{f}$ & 20,44 A b \\
\hline SMA502-1 & $40,38 \mathrm{~A}$ & 15,49 B $\mathrm{c}$ & $54,11 \mathrm{~B} \quad \mathrm{~d}$ & $62,53 \mathrm{~A} \quad \mathrm{~d}$ & $20,25 \mathrm{~A} \quad \mathrm{f}$ & $18,08 \mathrm{~A} \quad \mathrm{~d}$ \\
\hline SMA505-7 & $52,07 \mathrm{~A}$ & $16,62 \mathrm{~B}$ с & 58,65 B c & $67,10 \mathrm{~A} \quad \mathrm{c}$ & $22,62 \mathrm{~A} \quad \mathrm{~d}$ & 19,65 B с \\
\hline Média & 26,08 & 20,59 & 62,20 & 65,98 & 23,31 & 20,75 \\
\hline CV\% & 10,58 & 8,02 & 3,14 & 2,19 & 2,33 & 4,21 \\
\hline
\end{tabular}

${ }^{1}$ Escala que varia do preto ao branco, os valores mais altos indicam coloração mais clara.

${ }^{2}$ Médias de clones, pelo teste de Scott-Knott (letra minúscula), e de épocas de cultivo, pelo teste de Tukey (letra maiúscula), não seguidas pela mesma letra diferem a $5 \%$ de probabilidade de erro.

os demais clones apresentaram cor aceitável de chips (55 $\left.\geq L^{*} \leq 70\right)$ (COLEMAN, 2004), tanto nas condições de cultivo de primavera, quanto de outono (Tabela 1). As condições de cultivo de primavera promoveram o escurecimento de chips dos clones SMA516-2, SMA514-8, SMA520-5, SMA502-1 e SMA505-7. Apesar de os teores de açúcares redutores estarem normalmente correlacionados com os valores de cor de chips(MELO, 1997; COELHO, et al., 1999; FELTRAN et al., 2004), altos teores de açúcares redutores produziram chips com cor aceitável, o que pode ser atribuído à baixa concentração de polifenóis totais (ZORZELLA et al., 2003; FREITAS et al., 2006). Isso poderia, em parte, explicar a diferença de valores de cor de chips entre os clones SMA514-8 e SMA505-3 no cultivo de outono. Ambos os clones apresentaram teores muito próximos de açúcares redutores. No entanto, o clone SMA514-8 apresentou um valor de cor de chips de 69,99, enquanto o clone SMA505-3 apresentou um valor de 63,57.
Com exceção dos clones SMA505-3 no cultivo de primavera e SMA513-2, SMA506-4, SMA5173, Asterix, SMA501-1 e SMA505-7 no outono, todos os demais apresentaram valores de matéria seca superiores a $20 \%$, o que é considerado como de boa qualidade para processamento (Tabela 1). Embora a época de cultivo não tenha afetado a matéria seca dos clones SMA508-2, SMA503-1, SMA506-4, SMIJ461-1, SMA514-8, SMA517-2, SMA505-3, SMA505-2, SMA504-2 e SMA502-1, o cultivo de primavera apresentou em média 2,56\% mais matéria seca em relação ao outono. Maiores teores de matéria seca dos tubérculos aumentam o rendimento e a textura (crocância) do produto processado, reduzindo a absorção de gordura durante a fritura (FELTRAN et al., 2004; OLIVEIRA et al., 2006).

A radiação solar, a temperatura média do ar e o fotoperíodo são crescentes durante a primavera e decrescentes durante o outono no Rio Grande do Sul (BISOGNIN et al., 2008), fazendo com que as condições 
Tabela 2 - Teor de amido na matéria seca e proporção de amilose no amido de tubérculos de clones de batata produzidos na primavera e no outono. Santa Maria, RS, 2007.

\begin{tabular}{|c|c|c|c|c|}
\hline \multirow{2}{*}{ Clones } & \multicolumn{2}{|c|}{------------Amido (mg g de $\mathrm{MS}^{-1}$ ) ------------- } & \multicolumn{2}{|c|}{----------------Amilose (\%)--------------- } \\
\hline & Primavera & Outono & Primavera & Outono \\
\hline SMA517-2 & $825,26 \mathrm{~A} \mathrm{a}^{1}$ & $666,79 \mathrm{~B} \mathrm{a}$ & $27,61 \mathrm{~A} \quad \mathrm{c}$ & $22,08 \mathrm{~A}$ \\
\hline SMA508-2 & $809,10 \mathrm{~A} \mathrm{a}$ & 631,88 B b & $36,12 \mathrm{~A} \mathrm{a}$ & 28,44 B \\
\hline SMA501-1 & 771,39 A b & 569,59 B c & $22,85 \mathrm{~A} \quad \mathrm{~d}$ & 26,94 A \\
\hline SMA513-2 & 769,37 A b & $625,72 \mathrm{~B}$ b & $32,23 \mathrm{~A} \quad \mathrm{~b}$ & $24,36 \mathrm{~B}$ \\
\hline Asterix & 753,88 A b & 560,69 B c & $31,62 \mathrm{~A}$ b & 29,66 A b \\
\hline SMIJ461-1 & $742,44 \mathrm{~A} \quad \mathrm{c}$ & $514,14 \mathrm{~B} \quad \mathrm{~d}$ & $32,57 \mathrm{~A}$ b & 30,69 A b \\
\hline SMA503-1 & 739,07 A c & 589,44 B c & $32,17 \mathrm{~A}$ b & $26,85 \mathrm{~A} \quad \mathrm{C}$ \\
\hline SMA514-8 & 739,07 A c & 630,51 B b & $28,57 \mathrm{~A} \quad \mathrm{C}$ & $23,33 \mathrm{~A}$ \\
\hline SMA502-1 & $732,34 \mathrm{~A} \quad \mathrm{c}$ & 658,58 B a & $28,42 \mathrm{~A} \quad \mathrm{c}$ & $22,18 \mathrm{~A}$ \\
\hline SMA517-3 & 728,97 A c & $538,78 \mathrm{~B} \quad \mathrm{~d}$ & $31,86 \mathrm{~A} \quad \mathrm{~b}$ & $33,45 \mathrm{~A} \mathrm{a}$ \\
\hline SMA505-7 & $726,95 \mathrm{~A} \quad \mathrm{c}$ & 622,98 B b & $28,51 \mathrm{~A} \quad \mathrm{C}$ & $25,26 \mathrm{~A}$ \\
\hline SMA504-2 & $705,40 \mathrm{~A} \quad \mathrm{~d}$ & 629,15 B b & $35,43 \mathrm{~A} \mathrm{a}$ & 24,93 B \\
\hline SMA506-4 & $704,39 \mathrm{~A} \quad \mathrm{~d}$ & 583,28 B c & $30,31 \mathrm{~A} \quad \mathrm{c}$ & $26,10 \mathrm{~A} \quad \mathrm{~d}$ \\
\hline SMA519-1 & $701,17 \mathrm{~A} \quad \mathrm{~d}$ & $529,88 \mathrm{~B} \quad \mathrm{~d}$ & $35,24 \mathrm{~A} \mathrm{a}$ & 29,65 A $\quad b$ \\
\hline SMA505-2 & $694,63 \mathrm{~A} \quad \mathrm{~d}$ & 630,51 B b & $35,18 \mathrm{~A} \mathrm{a}$ & $28,12 \mathrm{~A} \quad \mathrm{c}$ \\
\hline SMA520-5 & $685,40 \mathrm{~A} \quad \mathrm{~d}$ & $544,94 \mathrm{~B} \quad \mathrm{~d}$ & $29,40 \mathrm{~A} \quad \mathrm{C}$ & 30,93 A b \\
\hline SMA505-3 & $683,18 \mathrm{~A} \quad \mathrm{~d}$ & 619,56 B b & $34,75 \mathrm{~A} \mathrm{a}$ & $27,85 \mathrm{~A}$ \\
\hline SMA516-2 & $668,03 \mathrm{~A} \quad \mathrm{e}$ & 600,39 B b & $30,55 \mathrm{~A} \quad \mathrm{c}$ & $27,49 \mathrm{~A}$ \\
\hline SMA508-4 & $667,35 \mathrm{~A} \quad \mathrm{e}$ & 608,61 B b & $37,84 \mathrm{~A} \mathrm{a}$ & 28,59 B \\
\hline SMA514-11 & $650,86 \mathrm{~A}$ e & 669,53 А а & $31,56 \mathrm{~A} \quad \mathrm{~b}$ & $24,03 \mathrm{~A} \quad \mathrm{~d}$ \\
\hline SMA514-10 & $650,58 \mathrm{~A} \quad \mathrm{e}$ & $661,32 \mathrm{~A}$ a & $31,47 \mathrm{~A} b$ & $27,06 \mathrm{~A}$ \\
\hline Média & 721,37 & 604,11 & 31,63 & 27,05 \\
\hline CV(\%) & 2,08 & 3,00 & 8,65 & 5,54 \\
\hline
\end{tabular}

${ }^{1}$ Médias de clones, pelo teste de Scott-Knott (letra minúscula), e de épocas de cultivo, pelo teste de Tukey (letra maiúscula), não seguidas pela mesma letra diferem a 5\% de probabilidade de erro.

climáticas de primavera sejam mais propícias para o cultivo da batata do que as de outono (FONTES \& FINGER, 1999). As condições contrastantes de primavera e outono pouco afetam o desenvolvimento das plantas de batata, mas a menor disponibilidade e eficiência de utilização da radiação solar no outono afetam, tanto a produtividade, quanto o tamanho dos tubérculos produzidos (BISOGNIN et al., 2008). Portanto, as condições de outono são menos favoráveis ao acúmulo de matéria seca nos tubérculos, o que é confirmado pelos resultados encontrados. Enquanto os teores mais elevados de matéria seca dos tubérculos são alcançados um pouco antes da senescência total da planta, os menores teores de açúcares redutores são alcançados somente quando as hastes encontram-se totalmente secas (MELO, 1997). Assim, os teores relativamente altos de açúcares redutores encontrados podem estar associados também a certo grau de imaturidade dos tubérculos, devido à morte prematura da parte aérea das plantas, o que poderia explicar esses teores elevados. Dessa forma, o momento da colheita deve ser considerado na seleção de clones de batata com baixos teores de açúcares redutores, principalmente quando os tubérculos são colhidos ainda imaturos.

As distintas condições entre os cultivos podem explicar os maiores teores de amido encontrados em tubérculos produzidos durante a primavera, que resultaram em uma média de amido na matéria seca 117,3 $\mathrm{mg} \mathrm{g}^{-1}$ superior a do cultivo de outono (Tabela 2), o que concorda com os resultados de FREITAS et al. (2006). Como o amido é o principal componente da matéria seca dos tubérculos (FONTES \& FINGER, 2000), qualquer fator que leve a um menor acúmulo também resultará em menores teores de matéria seca. Dos 21 clones avaliados neste trabalho, as condições de cultivo de primavera e outono não afetaram o teor de matéria seca de 10 clones; porém, o teor de amido não foi afetado em apenas dois clones. Os teores de amilose variaram no cultivo de primavera de 22,8 a $37,8 \%$, 
respectivamente, para os clones SMA501-1 e SMA5084, e no cultivo de outono de 22,1 a 33,4\%, respectivamente, para os clones SMA517-2 e SMA5173. Os teores de amilose encontrados foram superiores à média de 20\% obtida por LEONEL (2005). No entanto, o estádio de desenvolvimento da planta (LEONEL, 2005) e as condições de cultivo (FRANCO et al., 2002; NODA et al., 2004) podem afetar a relação entre amido e amilose. Os teores de amilose dos clones SMA508-2, SMA513-2, SMA504-2 e SMA508-4 foram maiores no cultivo de primavera do que de outono.

No melhoramento genético de batata, a seleção de clones que combinem o maior número de caracteres desejáveis muitas vezes é dificultada pelo grande número de clones e de caracteres avaliados. Dessa forma, a utilização de um índice de classificação dos clones para cada caráter avaliado, bem como o somatório dos índices de cada clone para todos os caracteres considerados, pode auxiliar na identificação de clones superiores (COSTA et al., 2004). A seleção baseada no somatório dos índices obtidos no cultivo de primavera e de outono resultou na identificação de diferentes grupos de clones, ou seja, os clones
SMA503-1, SMA508-2, SMA508-4, SMA513-2 e SMA519-1 foram selecionados na primavera e os clones SMA501-1, SMA508-2, SMA508-4, SMA514-8, SMA519-1 e SMA520-5, no outono, por apresentarem a melhor combinação de caracteres de qualidade quando comparados à melhor testemunha (SMIJ4611) (Tabela 3). Isso mostra que as condições de cultivo de primavera e de outono resultam na seleção de diferentes clones, ou seja, existem clones mais bem adaptados às condições de cultivo de uma época do que da outra. Para as condições de cultivo do Rio Grande do Sul, é necessária a identificação de clones que apresentam bom desempenho, tanto no cultivo de primavera, quanto de outono. Neste trabalho, somente os clones SMA508-2, SMA508-4 e SMA519-1 apresentaram desempenho superior à melhor testemunha em ambas as épocas de cultivo.

A seleção dos clones no cultivo de primavera proporcionou maiores ganhos médios de seleção (73,6\%) de todos os caracteres em comparação ao cultivo de outono (17,0\%) (Tabela 4). Em soja, COSTA et al. (2004) obtiveram maiores ganhos de seleção para a maioria dos caracteres do que os obtidos

Tabela 3 - Índices de classificação de 21 clones de batata produzidos na primavera e no outono para os cinco caracteres de qualidade para processamento avaliados na primavera e no outono. Santa Maria, RS, 2007.

\begin{tabular}{|c|c|c|c|c|c|c|c|c|c|c|c|c|}
\hline \multirow{2}{*}{ Clones } & \multicolumn{6}{|c|}{-----------------------------Primavera--------------------------- } & \multicolumn{6}{|c|}{ 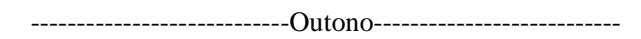 } \\
\hline & $\mathrm{AR}^{1}$ & $\mathrm{CC}^{1}$ & $\mathrm{MS}^{1}$ & $\mathrm{AM}^{1}$ & $\mathrm{AL}^{1}$ & Total & $\mathrm{AR}^{1}$ & $\mathrm{CC}^{1}$ & $\mathrm{MS}^{1}$ & $\mathrm{AM}^{1}$ & $\mathrm{AL}^{1}$ & Total \\
\hline SMA508-2 & $3^{2}$ & 4 & 5 & 2 & 2 & $16^{*}$ & 21 & 6 & 1 & 5 & 7 & $40^{*}$ \\
\hline SMA513-2 & 1 & 5 & 4 & 4 & 8 & $22 *$ & 4 & 1 & 16 & 9 & 17 & 47 \\
\hline SMA519-1 & 2 & 2 & 1 & 14 & 4 & $23^{*}$ & 1 & 10 & 2 & 20 & 5 & $38 *$ \\
\hline SMA503-1 & 4 & 7 & 11 & 7 & 9 & $38 *$ & 15 & 8 & 10 & 14 & 13 & 60 \\
\hline SMA508-4 & 16 & 1 & 3 & 19 & 1 & $40 *$ & 19 & 4 & 3 & 12 & 6 & $44^{*}$ \\
\hline SMIJ461-1 & 9 & 12 & 10 & 6 & 7 & 44 & 12 & 3 & 8 & 21 & 3 & 47 \\
\hline SMA501-1 & 5 & 10 & 8 & 3 & 21 & 47 & 2 & 9 & 6 & 16 & 12 & $45^{*}$ \\
\hline SMA514-10 & 7 & 9 & 2 & 21 & 13 & 52 & 18 & 16 & 5 & 3 & 11 & 53 \\
\hline Asterix & 10 & 15 & 13 & 5 & 11 & 54 & 13 & 17 & 18 & 17 & 4 & 69 \\
\hline SMA514-11 & 14 & 3 & 7 & 20 & 12 & 56 & 10 & 5 & 13 & 1 & 18 & 47 \\
\hline SMA506-4 & 6 & 6 & 17 & 13 & 15 & 57 & 20 & 13 & 19 & 15 & 14 & 81 \\
\hline SMA517-2 & 13 & 14 & 9 & 1 & 20 & 57 & 11 & 12 & 7 & 2 & 21 & 53 \\
\hline SMA505-2 & 18 & 8 & 16 & 15 & 5 & 62 & 14 & 15 & 11 & 6 & 8 & 54 \\
\hline SMA514-8 & 12 & 11 & 14 & 8 & 17 & 62 & 5 & 2 & 4 & 7 & 19 & $37 *$ \\
\hline SMA517-3 & 8 & 17 & 18 & 10 & 10 & 63 & 9 & 20 & 21 & 19 & 1 & 70 \\
\hline SMA520-5 & 15 & 13 & 6 & 16 & 16 & 66 & 3 & 11 & 9 & 18 & 2 & $43^{*}$ \\
\hline SMA504-2 & 19 & 19 & 19 & 12 & 3 & 72 & 17 & 21 & 14 & 8 & 16 & 76 \\
\hline SMA516-2 & 11 & 20 & 12 & 18 & 14 & 75 & 16 & 14 & 15 & 13 & 10 & 68 \\
\hline SMA505-3 & 17 & 18 & 21 & 17 & 6 & 79 & 6 & 18 & 12 & 11 & 9 & 56 \\
\hline SMA505-7 & 21 & 16 & 15 & 11 & 18 & 81 & 8 & 7 & 17 & 10 & 15 & 57 \\
\hline SMA502-1 & 20 & 21 & 20 & 9 & 19 & 89 & 7 & 19 & 20 & 4 & 20 & 70 \\
\hline
\end{tabular}

${ }^{1} \mathrm{AR}=$ açúcares Redutores; $\mathrm{CC}$ = coloração de chips; $\mathrm{MS}$ = matéria seca; $\mathrm{AD}$ = amido; $\mathrm{AL}=$ amilose.

${ }^{2}$ Índices de classificação dos clones para cada um dos caracteres conforme MULAMBA \& MOCK (1978).

* Clones com menores somas de índices do que a melhor testemunha (SMIJ461-1). 
Expressão dos caracteres e seleção de clones de batata nas condições de cultivo de primavera e outono.

Tabela 4 - Média dos clones selecionados (MCS) e originais (MCO) e ganhos indiretos de seleção (GS) com a soma dos índices de caracteres avaliados em tubérculos de 21 clones de batata produzidos na primavera e no outono. Santa Maria, RS, 2007.

\begin{tabular}{|c|c|c|c|c|c|c|c|c|}
\hline \multirow[t]{2}{*}{ Caracteres } & \multicolumn{4}{|c|}{-----------------------Primavera---------------------- } & \multicolumn{4}{|c|}{----------------------Outono---------------------- } \\
\hline & $\mathrm{MCS}^{1}$ & $\mathrm{MOC}^{2}$ & $\mathrm{GS}^{3}$ & GS\% ${ }^{4}$ & $\mathrm{MCS}^{1}$ & MOC $^{2}$ & $\mathrm{GS}^{3}$ & GS\% ${ }^{4}$ \\
\hline Açúcares redutores & 17,81 & 26,08 & 8,27 & $-46,43$ & 19,82 & 20,59 & 0,77 & $-3,88$ \\
\hline Coloração dos chips & 66,97 & 62,20 & 4,77 & 7,67 & 67,73 & 65,98 & 1,75 & 2,65 \\
\hline Matéria seca & 25,08 & 23,31 & 1,77 & 7,59 & 22,21 & 20,75 & 1,46 & 7,04 \\
\hline Amido & 37,21 & 721,37 & 15,84 & 2,19 & 585,90 & 604,11 & $-18,21$ & --- \\
\hline Amilose & 34,72 & 31,63 & 3,09 & 9,77 & 27,98 & 27,05 & 0,93 & 3,44 \\
\hline Total & & & & 73,65 & & & & 17,01 \\
\hline
\end{tabular}

${ }^{1}$ MCS= média dos clones selecionados;

${ }^{2} \mathrm{MOC}=$ média original dos clones;

${ }^{3} \mathrm{GS}=$ ganho de seleção;

${ }^{4}$ GS\% = ganho de seleção expresso em porcentagem. Conforme tabela 3, os clones selecionados na primavera foram SMA503-1, SMA508-

2, SMA508-4, SMA513-2 e SMA519-1 e, no outono, SMA501-1, SMA508-2, SMA508-4, SMA514-8, SMA519-1 e SMA520-5.

pela seleção direta, sendo as estimativas de ganhos totais superiores quando utilizaram o índice de MULAMBA \& MOCK (1978), também usado neste trabalho. Dessa forma, a utilização de índices de classificação dos clones mostra-se vantajosa em relação à seleção direta, uma vez que possibilita ganhos mais bem distribuídos em todos os caracteres avaliados. Além disso, o melhorista pode especificar diferentes pesos para o índice de cada caráter avaliado, fazendo com que clones com melhores índices em caracteres mais importantes sejam beneficiados no somatório final, o que pode ser explorado em trabalhos futuros.

Quando comparados os cultivos, observase que a identificação dos clones com potencial de processamento na forma de chips é muito mais eficaz quando a seleção é realizada no cultivo de primavera, pois um ganho similar de seleção foi obtido somente para o teor de matéria seca (GS de 7,6\% na primavera e de 7,4\% no outono) (Tabela 4). Além disso, o maior ganho de seleção foi obtido no cultivo de primavera para a seleção de clones com baixos teores de açúcares redutores (GS de 46,4\%), que é o principal fator que determina a cor de chips (FELTRAN et al., 2004). A combinação de qualidade de processamento com produtividade e adaptação às condições de cultivo contribuirá para a redução da dependência por cultivares estrangeiras e da importação de batata préfrita e congelada para atender o mercado brasileiro de batata processada.

\section{CONCLUSÕES}

A seleção de clones para processamento industrial deve ser realizada no cultivo de primavera, quando as condições ambientais são mais favoráveis para a expressão dos caracteres de qualidade de tubérculo. Os clones SMA508-2, SMA508-4 e SMA5191 apresentam a melhor combinação de caracteres associados à qualidade de processamento nas condições de cultivo de primavera e outono do Rio Grande do Sul.

\section{AGRADECIMENTOS}

Ao Conselho Nacional de Desenvolvimento Científico e Tecnológico (CNPq), pelo financiamento parcial e pela concessão de bolsas de pesquisa e iniciação científica, e à Fundação de Amparo à Pesquisa do Estado do Rio Grande do Sul (FAPERGS), pela concessão de bolsa de iniciação científica.

\section{REFERÊNCIAS}

BISOGNIN, D.A. Recomendações técnicas para o cultivo da batata no Rio Grande do Sul e Santa Catarina. Santa Maria: UFSM, 1996. 64p.

BISOGNIN, D.A. et al. Desenvolvimento e rendimento de clones de batata na primavera e no outono. Pesquisa Agropecuária Brasileira, v.43, n.6, p.699-705, 2008. Disponível em: <http:// www.scielo.br/scielo.php?script=sci_pdf\&pid=S0100204X2008000600005\&lng=en\&nrm=iso\&tlng=pt > . Doi: 10.1590/ S0100-204X2008000600005.

BRODY, J. Pointers on potatoes: potential of processed potatoes on the increase; product variables and process factors discussed; varieties check listed. Food Engineer, v.47, n.9, p.124-132, 1969.

COELHO, A.H.R. et al. Qualidade de batata (Solanum tuberosum L.) para fritura, em função dos níveis de açúcares redutores e amido, durante o armazenamento refrigerado e à temperatura ambiente com atmosfera modificada. Ciência e Agrotecnologia, v.23, n.4, p.899-910, 1999.

COLEMAN, W.K. Comparative performance of the $\mathrm{L}^{*} \mathrm{a}^{*} \mathrm{~b}$ * color space and North American color charts for determining 
chipping quality in tubers of potato (Solanum tuberosum L.). Canadian Journal of Plant Science, v.84, n.1, p.291-298, 2004.

COSTA, M.M. et al. Ganho genético por diferentes critérios de seleção em populações segregantes de soja. Pesquisa Agropecuária Brasileira, v.39, n.11, p.1095-1102, 2004. Disponível em: <http:/ /www.scielo.br/scielo.php?script=sci_arttext\&pid=S0100204X2004001100007\&lng=en\&nrm=iso>. Doi: 10.1590/S0100204X2004001100007.

CRUZ, C.D.; REGAZZI, A.J. Modelos biométricos aplicados ao melhoramento genético. Viçosa: UFV, 1997. 390p.

FELTRAN, J.C. et al. Technological quality and utilization of potato tubers. Scientia Agricola, v.61, n.6 p.598-603, 2004. Disponível em: <http://www.scielo.br/scielo.php?pid=S010390162004000600006\&script $=$ sci_arttext $>$. Doi: 10.1590/ S0103-90162004000600006.

FONTES, P.C.R.; FINGER, F.L. Dormência dos tubérculos, crescimento da parte aérea e tuberização da batateira. Informe Agropecuário, v.20, n.197, p.24-29, 1999.

FONTES, P.C.R.; FINGER, F.L. Pós-colheita do tubérculo de batata. Viçosa: UFV, 2000. 32p.

FRANCO, C.M.L. et al. Propriedades gerais do amido. Campinas: Fundação Cargill, 2002. 224p.

FREITAS, S.T. et al. Qualidade para processamento de clones de batata cultivados durante a primavera e outono no Rio Grande do Sul. Ciência Rural, v.36, n.1, p.80-85, 2006. Disponível em: $<$ ht tp://www.scielo.br/scielo.php?pid=S 0103 $84782006000100012 \&$ script=sci_arttext $>$. Doi: 10.1590/S010384782006000100012 .

GILBERT, G.A.; SPRAGG, S.P. Iodometric determination of amylose. In: WHISTLER, R.L. (Ed.). Methods in carbohydrate chemistry starch. New York: Academic, 1964. V.4, p.168-169.

HERTOG, M.L.A.T.M. et al. The effects of temperature and senescence on the accumulation of reducing sugars during storage of potato (Solanum tuberosum L.) tubers: A mathematical model. Postharvest Biology and Technology, v.10, p.67-79, 1997. Disponível em: <www.ingentaconnect.com/els/09255214/1997/ 00000010/00000001/art87276>. Doi: 10.1016/S09255214(97)87276-6

LEONEL, M. Processamento de batata: fécula, flocos, produtos de extrusão. In: SEMINÁRIO MINEIRO SOBRE PROCESSAMENTO DE BATATAS, Pouso Alegre, Minas Gerais. Anais... Pouso Alegre: EPAMIG, 2005. CD.

LONG, A.R.; CHISM, G.W. Physical and chemical methods of evaluation foods. Acessado em 8 jun. 2004. Online. Disponível em: http://food.oregonstate.edu/research/test/ reducing.html.

MELO, P.E. Aptidão de cultivares de batata para consumo in natura e para processamento. In: GUEDES, J.V.C. Seminário de atualização na cultura da batata. Santa Maria: Sociedade de Agronomia de Santa Maria: UFSM, 1997. p.27-38.

MULAMBA, N.N.; MOCK, J.J. Improvement of yield potential of the method Eto Blanco maize (Zea mays L.) population by breeding for plant traits. Egyptian Journal of Genetics and Citology, v.7, n.1, p.40-51, 1978.

NODA, T. et al. The effect of harvest dates on the starch properties of various cultivars. Food Chemistry, v.86, n.1, p.119-125, 2004. Disponível em: < http://www.sciencedirect.com/ science?_ob=ArticleURL\&_udi=B6T6R-4B4RSHP $7 \&$ \&user $=10 \&$ \&_coverDate $=06 \% 2$ F $30 \% 2$ F $2004 \&$ \&doc $=$ $1 \&$ _fmt $=$ high \&_orig =browse \&_sort $=$ d \& view $=$ c $\&$ _ a c ct $=$ C $000050221 \&$ _version $=1 \&$ \&_urlVersion $=0$ \&_userid=10\&md5=4c77179def9e5e8ef721eeb72c83bd17>. Doi:10.1016/j.foodchem.2003.09.035.

OLIVEIRA, V.R. et al. Qualidade de processamento de tubérculos de batata produzidos sob diferentes disponibilidades de nitrogênio. Ciência Rural, v.36, n.2, p.660-663, 2006. Disponível em: $<$ ht t p: / / w w w.scielo.br/scielo.php?pid=S 0103 $84782006000200048 \&$ script $=$ sci_arttext\&tlng $=e n>$. Doi: 10.1590/S0103-84782006000200048.

SILVA, A.C.F. Batata: alguns aspectos importantes. Agropecuária Catarinense, v.4, n.4, p.38-41, 1991.

ZORZELLA, C.A. et al. Caracterização física, química e sensorial de genótipos de batata processados na forma de chips. Brazilian Journal of Food Technology, v.6, n.1, p.15-24, 2003. 